\title{
A Novel Un-differenced PPP-RTK Concept
}

\author{
B.C. Zhang $^{1}$, P. J.G. Teunissen ${ }^{2,3}$ and D. Odijk ${ }^{2}$ \\ (1: Institute of Geodesy and Geophysics, Chinese Academy of Sciences, Wuhan, China) \\ (2: GNSS Research Centre, Curtin University of Technology, Perth, Australia) \\ (3: Delft Institute of Earth Observation and Space Systems, Delft University of Technology, The \\ Netherlands) \\ (E-mail: b.zhang@whigg.ac.cn; p.teunissen@curtin.edu.au)
}

\begin{abstract}
In this contribution, a novel un-differenced (UD) (PPP-RTK) concept, i.e. a synthesis of Precise Point Positioning and Network-based Real-Time Kinematic concept, is introduced. In the first step of our PPP-RTK approach, the UD GNSS observations from a regional reference network are processed based upon re-parameterised observation equations, corrections for satellite clocks, phase biases and (interpolated) atmospheric delays are calculated and provided to users. In the second step, these network-based corrections are used at the user site to restore the integer nature of his UD phase ambiguities, which makes rapid and high accuracy user positioning possible. The proposed PPP-RTK approach was tested using two GPS CORS networks with inter-station distances ranging from 60 to 100 $\mathrm{km}$. The first test network is the northern China CORS network and the second is the Australian Perth CORS network. In the test of the first network, a dual-frequency PPP-RTK user receiver was used, while in the test of the second network, a low-cost, single-frequency PPP-RTK user receiver was used. The performance of fast ambiguity resolution and the high accuracy positioning of the PPP-RTK results are demonstrated.
\end{abstract}

\section{KEY WORDS}

1. GNSS. 2. PPP-RTK. 3. Integer Ambiguity Resolution. 4. Satellite Phase Bias.

1. INTRODUCTION. Precise Point Positioning (PPP) and Network-based Real-Time Kinematic (NRTK) are two representative techniques for high accuracy Global Navigation Satellite System (GNSS)-based positioning (Kouba and Heroux, 2001; Wuebbena et al, 2005). Based on observations from a stand-alone GNSS receiver and the IGS precise orbit and clock products, PPP positioning accuracy can reach cm- to dm-levels for static or kinematic applications (Bree et al, 2009). However, due to the fact that PPP-based solutions use real-valued float ambiguities, i.e. non-integer un-differenced (UD) phase ambiguities, long convergence times are experienced (Zumberge et al, 1997). NRTK does not have this drawback as observations from one of the (virtual) network reference stations are used by the NRTK user and the virtual station's observations contain the error corrections that are derived from the reference network. This allows the NRTK users to perform fast Integer Ambiguity Resolution (IAR) and realise positioning with accuracy at the cm-level using only a few epochs of data (Vollath et al, 2000; Wu et al, 2008; Odijk et al, 2010).

A novel UD PPP-RTK concept, which is a synthesis of PPP and NRTK, is proposed and analyzed in this paper, and some of its test results and performance assessment are demonstrated. There are two differences between our PPP-RTK concept and those existing ones (for example, Wuebbena et al, 2005; Zhang et al, 2006; Geng et al, 2010; Li et al, 2010). First, the UD observations, rather than their ionosphere-free combinations, are used to directly provide the ionospheric corrections. Secondly, all the 
corrections can be obtained in one go, i.e. no step-wise processing procedures are required, thus the consistency amongst different types of corrections can be assured.

Two distinct parts in the implementation of our PPP-RTK approach are. First, the UD observations are processed at the network level. After eliminating the rank deficiencies with re-parameterization, or S-basis choice (De Jonge, 1998), the network parameters can be estimated in real-time with a Kalman-filter or recursive least squares. Once the network ambiguities are successfully resolved, the ambiguity-fixed (biased) satellite clocks, (biased) satellite phase biases, and the (interpolated) ionospheric delays for the user's location will be saved and ready for sending to the PPP users that are within the network region. Secondly, after correcting their observations with the network corrections, the PPP users can perform IAR and positioning just as they do in the case of NRTK.

In the following sections, we first derive the observation equations of our PPP-RTK approach and special attention is given to rank defects and parameter estimability. Subsequently, the performance of the PPP-RTK approach will be tested and its capability for fast IAR and high accuracy user positioning is demonstrated.

2. THE PPP-RTK CONCEPT. In this section, the full-rank observation equations of our PPP-RTK concept are given. This is done for data processing of both the network and the user sites. Although the work presented in this contribution only used single- and dual-frequency GPS data, the proposed PPP-RTK concept is also applicable to multi-frequency and multi-GNSS applications. In the following sub-sections, we firstly discuss the network observation equations and then the user observation equations.

2.1. Network Processing. For a receiver-satellite pair $r-s$, the UD carrier phase and code observation equations on frequency $j$ can be given as (Teunissen and Kleusberg, 1998):

$$
\begin{aligned}
& E\left(\varphi_{r, j}^{s}\right)=\rho_{r}^{s}-\mu_{j} I_{r}^{s}+\lambda_{j} N_{r, j}^{s}+\phi_{r, j}-\phi_{, j}^{s} \\
& E\left(p_{r, j}^{s}\right)=\rho_{r}^{s}+\mu_{j} I_{r}^{s}+b_{r, j}-b_{, j}^{s}
\end{aligned}
$$

where E(.) denotes the expectation operator, $\varphi_{r, j}^{s}$ and $p_{r, j}^{s}$ denote the phase and code observable, respectively, $\rho_{r}^{s}=l_{r}^{s}+\tau_{r}^{s}+d t_{r}-d t^{s}$ is the sum of all the frequency-independent items, $l_{r}^{s}$ is the receiver-satellite range, $\tau_{r}^{s}$ the slant tropospheric delay, $d t_{r}$ and $d t^{s}$ are the receiver and satellite clock errors respectively, $I_{r}^{s}$ is the (first-order) slant ionospheric delay on the L1 frequency $\left(\mu_{j}=\lambda_{j}^{2} / \lambda_{1}^{2}, \mu_{2} \approx 1.6469\right), N_{r, j}^{s}$ is the integer phase ambiguity, $\lambda_{j}$ the wavelength of frequency $j$, $\phi_{r, j}$ and $\phi_{, j}^{s}$ are the frequency-dependent phase biases, while $b_{r, j}$ and $b_{, j}^{s}$ are that of their code counterparts. Note that all the bias terms are in units of meters.

For the purpose of simplifying our equations in the following sections, the following assumptions for the network processing are made:

- The network consists of $n$ stations $(r=1, \cdots n)$ and all the stations track the same $m$ satellites $(s=1, \cdots m)$ on the L1 and L2 frequencies $(j=1,2)$; 
- The geometric range of the receiver to the satellite $l_{r}^{s}$ is known from the known position of the reference station and the satellite provided by the IGS precise orbit.

For practical purposes and the flexibility of our approach, we now discuss the approach for the following two cases: 1) the satellite clock information is provided externally, i.e. it is available to the users, and 2) the satellite clock information is not available, thus it must be provided by the regional network.

2.1.1. When Satellite Clocks Are Available. The satellite clocks given below are assumed known from an external source e.g. from IGS,

$$
d t_{I}^{s}=d t^{s}+\frac{\mu_{2}}{\mu_{2}-1} \cdot b_{11}^{s}-\frac{1}{\mu_{2}-1} \cdot b_{, 2}^{s}
$$

Then the code observation equation expressed in Equation (1) can be reformulated

$$
E\left(p_{r, j}^{s}-l_{r}^{s}+d t_{I}^{s}\right)=\tau_{r}^{s}+d \bar{t}_{r}+\mu_{j} \bar{I}_{r}^{s}
$$

In Equation (3), all the code biases originating from $p_{r, j}^{s}$ and $d t_{I}^{s}$ are absorbed by the estimable receiver clock and the slant ionospheric delays. Their re-parameterised forms are therefore given as:

$$
\left\{\begin{array}{l}
d \bar{t}_{r}=d t_{r}+\frac{\mu_{2}}{\mu_{2}-1} \cdot b_{r, 1}-\frac{1}{\mu_{2}-1} \cdot b_{r, 2} \\
\bar{I}_{r}^{s}=I_{r}^{s}-\frac{1}{\mu_{2}-1} \cdot\left(B^{s}-B_{r}\right)
\end{array}\right.
$$

where the $B^{s}=b_{, 2}^{s}-b_{, 1}^{s}$ and $B_{r}=b_{r, 2}-b_{r, 1}$ are the satellite and receiver Differential Code Biases (DCBs), respectively (Sardon and Zarraoa, 1997; Yuan and Ou, 2001).

Similarly, the network phase observation equations can be obtained after the same re-parameterisation:

$$
E\left(\varphi_{r, j}^{s}-l_{r}^{s}+d t_{I}^{s}\right)=\tau_{r}^{s}+d \bar{t}_{r}-\mu_{j} \bar{I}_{r}^{s}+\lambda_{j} N_{r, j}^{s}+\bar{\phi}_{r, j}-\bar{\phi}_{, j}^{s}
$$

Due to the opposite sign of the ionospheric group and phase delays, the code biases within $d \bar{t}_{r}$ and $\bar{I}_{r}^{s}$ cannot be cancelled as in the code observation expressed in Equation (3). Hence, the re-parameterised phase biases are given as:

$$
\left\{\begin{array}{l}
\bar{\phi}_{r, j}=\phi_{r, j}-\frac{\mu_{2} b_{r, 1}-b_{r, 2}}{\mu_{2}-1}+\frac{\mu_{j}}{\mu_{2}-1} B_{r} \\
\bar{\phi}_{, j}^{s}=\phi_{, j}^{s}-\frac{\mu_{2} b_{, 1}^{s}-b_{, 2}^{s}}{\mu_{2}-1}+\frac{\mu_{j}}{\mu_{2}-1} B^{s}
\end{array}\right.
$$

Due to the linear dependence between $N_{r, j}^{s}, \bar{\phi}_{r, j}$ and $\bar{\phi}_{, j}^{s}$, there would be a rank defect of $f(n+m)$ in the design matrix of Equation (5). One possible solution to this problem is to choose, per frequency, the phase biases of the first receiver (here $\bar{\phi}_{1, j}$ ), the ambiguities from the first satellite to all involved 
receivers (here $N_{r, j}^{1}$ ) and the ambiguities from the first receiver to all visible satellites (here ${ }_{1, j}^{s}$ ) as S-basis (Teunissen et al, 2010). Then the network phase equations can be expressed as:

$$
E\left(\varphi_{r, j}^{s}-l_{r}^{s}+d t_{I}^{s}\right)=\tau_{r}^{s}+d \bar{t}_{r}-\mu_{j} \bar{I}_{r}^{s}+\lambda_{j} N_{r 1, j}^{s 1}+\tilde{\phi}_{r, j}-\tilde{\phi}_{, j}^{s}
$$

where $\tilde{\phi}_{, j}^{s}=\bar{\phi}_{, j}^{s}-\bar{\phi}_{1, j}-\lambda_{j} N_{1, j}^{s}, \quad \tilde{\phi}_{r, j}=\bar{\phi}_{r 1, j}+\lambda_{j} N_{r 1, j}^{1}$ and $N_{r 1, j}^{s 1}=N_{r 1, j}^{s}-N_{r 1, j}^{1}$. Thus in Equation (6), we can find the integer double differenced (DD) ambiguities ${ }^{s 1, j}$.

To avoid possible additional rank defects, the slant tropospheric delays $\tau_{r}^{s}$ in both Equations (3) and (6) can be further parameterised:

$$
\tau_{r}^{s}=m f_{r}^{s} \cdot T_{r}
$$

where $T_{r}$ is the station-wise Zenith Tropospheric Delay (ZTD) and $m f_{r}^{s}$ is a mapping function.

To summarise, when the satellite clock information is available from an external source, the PPP-RTK network processing of the phase and code data can be based on the following set of full-rank, re-parameterised observation equations:

$$
\left\{\begin{array}{c}
E\left(p_{r, j}^{s}-l_{r}^{s}+d t_{I}^{s}\right)=m f_{r}^{s} \cdot T_{r}+d \bar{t}_{r}+\mu_{j} \bar{I}_{r}^{s} \\
E\left(\varphi_{r, j}^{s}-l_{r}^{s}+d t_{I}^{s}\right)=m f_{r}^{s} \cdot T_{r}+d \bar{t}_{r}-\mu_{j} \bar{I}_{r}^{s}+\lambda_{j} N_{r, j}^{s}+\bar{\phi}_{r, j}-\bar{\phi}_{, j}^{s}
\end{array}\right.
$$

2.1.2. When Satellite Clocks Are Unavailable. When the external satellite clocks are not available, they can be derived from a regional reference network. In this case, the network observation equations need to accommodate the additional unknowns and additional (near) rank defects. These rank defects are due to the linear dependence between the satellite clocks and receiver clocks, and the near linear dependence between the satellite clocks and the ZTD mapping functions. This problem can be solved by choosing the clock and ZTD of the first receiver to be the S-basis. Then the final full-rank observation equations of the network become:

$$
\left\{\begin{array}{l}
E\left(p_{r, j}^{s}-l_{r}^{s}\right)=m f_{r}^{s} \cdot \tilde{T}_{r}+d \tilde{t}_{r}-d \tilde{t}_{I}^{s}+\mu_{j} \bar{I}_{r}^{s} \\
E\left(\varphi_{r, j}^{s}-l_{r}^{s}\right)=m f_{r}^{s} \cdot \tilde{T}_{r}+d \tilde{t}_{r}-d \tilde{t}_{I}^{s}-\mu_{j} \bar{I}_{r}^{s}+\lambda_{j} N_{r 1, j}^{s 1}+\tilde{\phi}_{r, j}-\tilde{\phi}_{, j}^{s}
\end{array}\right.
$$

where $\tilde{T}_{r}=T_{r}-T_{1}$ is the estimable ZTDs and $d \tilde{t}_{I}^{s}=d t_{I}^{s}-d \bar{t}_{1}+m f_{1}^{s} \cdot T_{1}$ and $d \tilde{t}_{r}=d \bar{t}_{r}-d \bar{t}_{1}$ are the redefined satellite and receiver clocks, respectively.

The network corrections that are provided to the PPP users include $d \tilde{t}_{I}^{s}$ and $\tilde{\phi}_{, j}^{s}$, which are essential for the user's fast IAR in PPP and the interpolated $\bar{I}_{r}^{s}$ which also help to improve the performance of IAR (Yuan et al, 2008a; Yuan et al, 2008b; Li et al, 2010). Although not tested in this contribution, the ZTDs from the network processing method can be also used to facilitate the user's PPP-IAR. The network processing strategy presented above can be used in both real-time and post-processing modes. 
2.2. PPP Processing. In the previous section, it was discussed that the ionospheric delays, i.e. $\bar{I}_{r}^{s}$ in Equation (4), derived from the network processing need to be interpolated in the spatial domain for generating the ionospheric delay corrections at the PPP user's approximate position. Several interpolation methods can be used for this purpose and the Kriging method (Jarlemark and Emardson, 1998) was selected in this research. The covariance function selected for the use of this method is a simple linear function of the inter-station distance. The resulting interpolated ionospheric delays can be expressed as:

$$
\begin{aligned}
& \tilde{I}_{u}^{s}=\sum_{r=1}^{n} f_{r} \cdot \bar{I}_{r}^{s} \\
& E\left(\tilde{I}_{u}^{s}\right)=I_{u}^{s}-\frac{1}{\mu_{2}-1} \cdot\left(B^{s}-\tilde{B}_{u}\right)
\end{aligned}
$$

where $f_{r}$ is the coefficient of the interpolation, subscript $u$ denotes the user, $I_{u}^{s}$ is the ionospheric delay of the user receiver $u$ to the satellite $s, B^{s}$ is the satellite's DCB, which is free from the interpolation process and $\tilde{B}_{u}$ is the “interpolated” DCB for the PPP user.

After applying the network-based corrections and the elimination of rank defects, the linearised PPP-RTK observation equations become:

$$
\left\{\begin{array}{l}
E\left(p_{u, j}^{s}-l_{u, 0}^{s}+d \overline{\tilde{t}}_{I}^{s}\right)=\boldsymbol{\mu}_{u}^{s} \cdot \Delta x_{u}+m f_{u}^{s} \cdot \tilde{T}_{u}+d \bar{t}_{u}+\mu_{j} \bar{I}_{u}^{s} \\
E\left(\varphi_{u, j}^{s}-l_{u, 0}^{s}+d \overline{\tilde{t}}_{I}^{s}+\tilde{\phi}_{, j}^{s}\right)=\boldsymbol{\mu}_{u}^{s} \cdot \Delta x_{u}+m f_{u}^{s} \cdot \tilde{T}_{u}+d \bar{t}_{u}-\mu_{j} \bar{I}_{u}^{s}+\lambda_{j} N_{u 1, j}^{s}+\bar{\phi}_{u, j} \\
E\left(\tilde{I}_{u}^{s}\right)=\bar{I}_{u}^{s}-\frac{1}{\mu_{2}-1} \cdot \Delta B_{u}
\end{array}\right.
$$

Where ${ }_{u, 0}^{s}$ is the approximate geometric range, $\boldsymbol{\mu}_{u}^{s}$ is the unit vector of the geometric range from the satellite to the user receiver, $\Delta x_{u}$ denotes the vector increment of the receiver position and the forms of $\tilde{T}_{u}, d \bar{t}$ and $\bar{I}_{u}^{s}$ are similar to those in the network equations, see Equation (8). The term $\Delta B_{u}=\left(B_{u}-\tilde{B}_{u}\right)$ in Equation (10) stems from the difference between the interpolated ionospheric delays $\tilde{I}_{u}^{s}$ and the estimable ionospheric delays $\bar{I}_{u}^{s}$.

Obviously, in Equation (10) $N_{u 1, j}^{s}$ and $\quad \bar{\phi}_{u, j}$ are linear dependent, its resulting rank defect could be eliminated by choosing, per frequency, the first satellite's single differenced (SD) ambiguity $N_{u 1, j}^{1}$ as the S-basis. After $\lambda_{j} N_{u 1, j}^{s}+\bar{\phi}_{u, j}$ in Equation (10) is replaced with $\lambda_{j} N_{u 1, j}^{s 1}+\tilde{\phi}_{u, j}$, where $\tilde{\phi}_{u, j}=\bar{\phi}_{u, j}+\lambda_{j} N_{u 1, j}^{1}$, the full-rank observation equation for PPP users can be obtained. 
The source of satellite clock estimate $d \overline{\tilde{t}}_{I}^{s}$ in Equation (10) is either the IGS clock estimate $d t_{I}^{s}$ or the regional network-based satellite clock estimate $d \tilde{t}_{I}^{s}$, which depends on the strategy adopted in the network processing, as discussed in sections 2.1.1 and 2.1.2. Based upon both types of satellite clocks, the user's PPP-RTK observation equations can be cast into a unified frame as given by Equation (10). Note, however, that the interpretations of the estimable parameters $\tilde{T}_{u}$ and $d \bar{t}_{u}$ are different in the two cases: when provided from IGS, the results of $d t_{I}^{s}$, the $\tilde{T}_{u}$ and $d \bar{t}_{u}$ are in the IGS frame; while when calculated from the regional network, the estimates of $d \tilde{t}_{I}^{s}, \quad \tilde{T}_{u}$ and $d \bar{t}_{u}$ can only be relative to the $T_{1}$ and $d \bar{t}_{1}$ that have been absorbed by $d \tilde{t}_{I}^{s}$. In our case studies discussed in the next section, the satellite clock parameters are assumed to be unavailable and they were estimated from the regional network.

3. CASE STUDIES. Based on the two CORS networks, one in northern China and the other in Perth, Western Australia, our PPP-RTK approach was tested. The performance of the network processing and the fast IAR capability for both dual- and single-frequency PPP at the user's end are demonstrated.

3.1. Northern China CORS: Dual-frequency PPP. This CORS network is a medium-scale network consisting of 4 stations with inter-station distances ranging from 60 to 100 km, see Figure 1. Trimble GPS data on two frequencies: L1 and L2 (L1-L2-C1-P2) on 29th April 2009 with 30 sec sampling rate were collected.

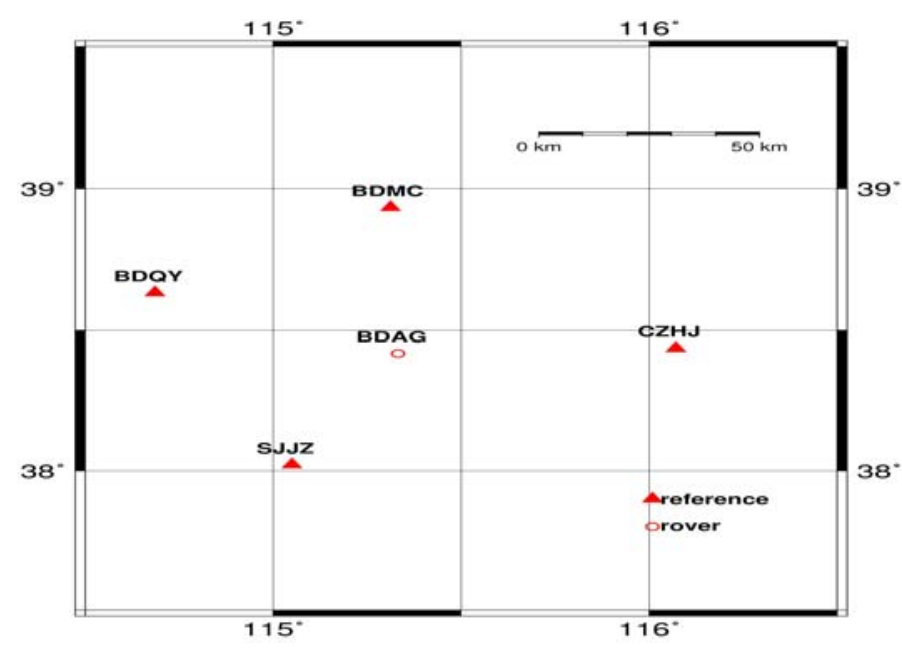

Figure 1. The configuration of the northern China CORS network consisting of 4 reference stations (triangles), and the BDAG station (circle) is the user station.

3.1.1. Network Processing Results. Our network processing strategy is characterised as follows. The standard deviations of the UD phase and code observations were set to $3 \mathrm{~mm}$ and $30 \mathrm{~cm}$, respectively. All the observations were weighted according to their elevation and the elevation cut-off angle of 5 degrees was used. A Kalman-filter was used for the real-time data processing. The residual ionospheric and ZTD 
tropospheric delays are modeled as a random walk process; the clock errors are modeled as white noise, while the DD integer ambiguities are treated as constants.

For both network-IAR and user-IAR, the LAMBDA method was used (Teunissen, 1995; Teunissen et al. 1996). For the validation of the integer ambiguity results, the fixed-failure rate FFRatio test was used (Teunissen and Verhagen, 2010). The epoch-wise full IAR was started after the filter's 10 epochs (5 min) initialisation. The float ambiguities corresponding to newly risen satellites were only considered for resolution after 60 epochs' (30 min) filtering. Panel (a) of Figure 2 shows the result of ambiguity resolution with a success rate of about $2873 / 2880 \approx 99.7 \%$. The epochs with wrongly fixed ambiguities are corresponding to the periods during which the satellites were frequently rising and setting, as revealed in panel (b).
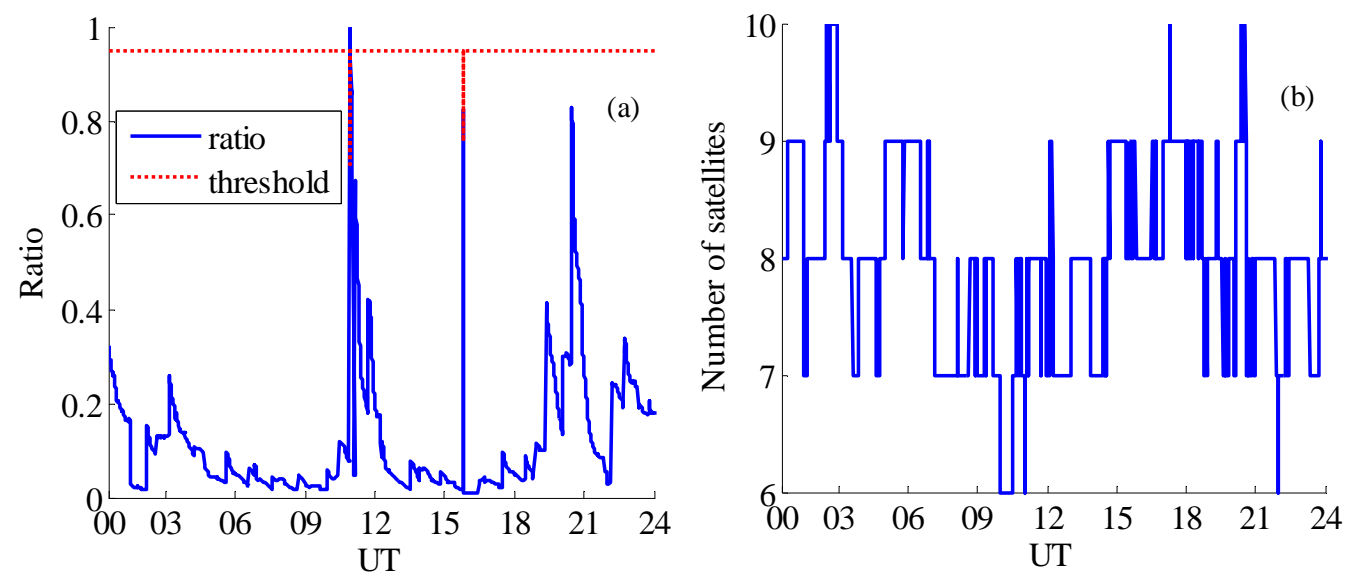

Figure 2. IAR results for the northern China CORS: panel (a) FFRatio test- and threshold values versus time; panel (b) number of tracked satellites versus time 

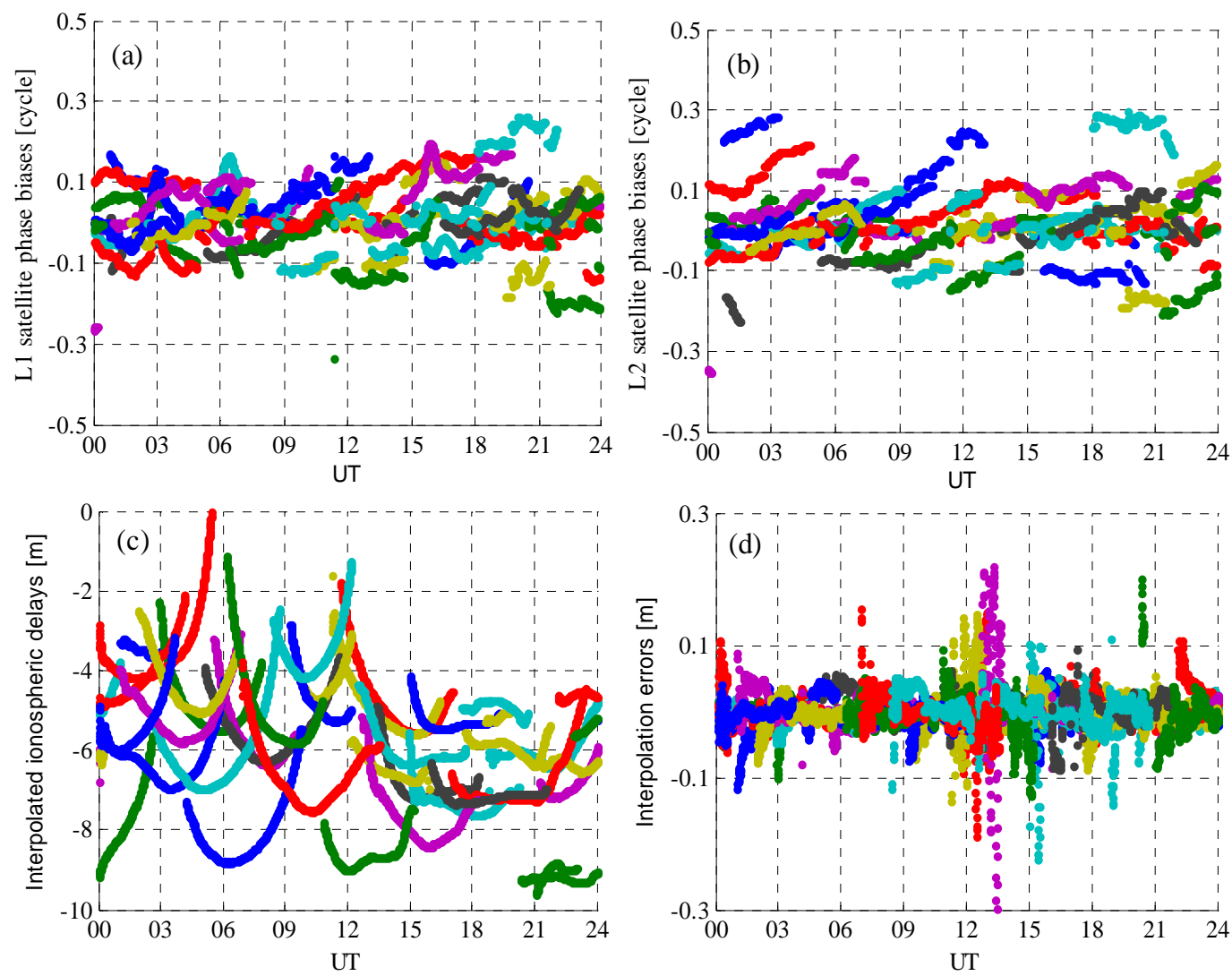

Figure 3. Processing results of the northern China CORS network: panels (a) \& (b): L1 \& L2 satellite phase biases (in units of cycles) respectively; panels (c) \& (d): interpolated ionospheric delays and their errors (in units of meters) respectively. Different colors correspond to different satellites

Figure 3 shows the network processing results. In the two upper panels of this figure, the estimated dual-frequency phase biases are shown for each satellite's continuous arc. The two lower panels of Figure 3 show the interpolated ionospheric delays and their errors/accuracy. These error estimates are obtained from comparing the interpolated delays with the reference values of the ionospheric delays at BDAG (user), which could be derived from post-processing the GPS data of the network together with BDAG. Note that most of the interpolation errors/accuracy at BDAG is less than $1 \mathrm{dm}$ for all the satellites, except those in some periods when the ionosphere was in disturbed conditions (i.e. 12:00-13:00 UT).

3.1.2. Dual-frequency PPP Result. During the static PPP processing, the epoch-wise interpolated ionospheric delays were used as pseudo observations, whose standard deviations were set to $1 \mathrm{dm}$ to account for the interpolation errors. 

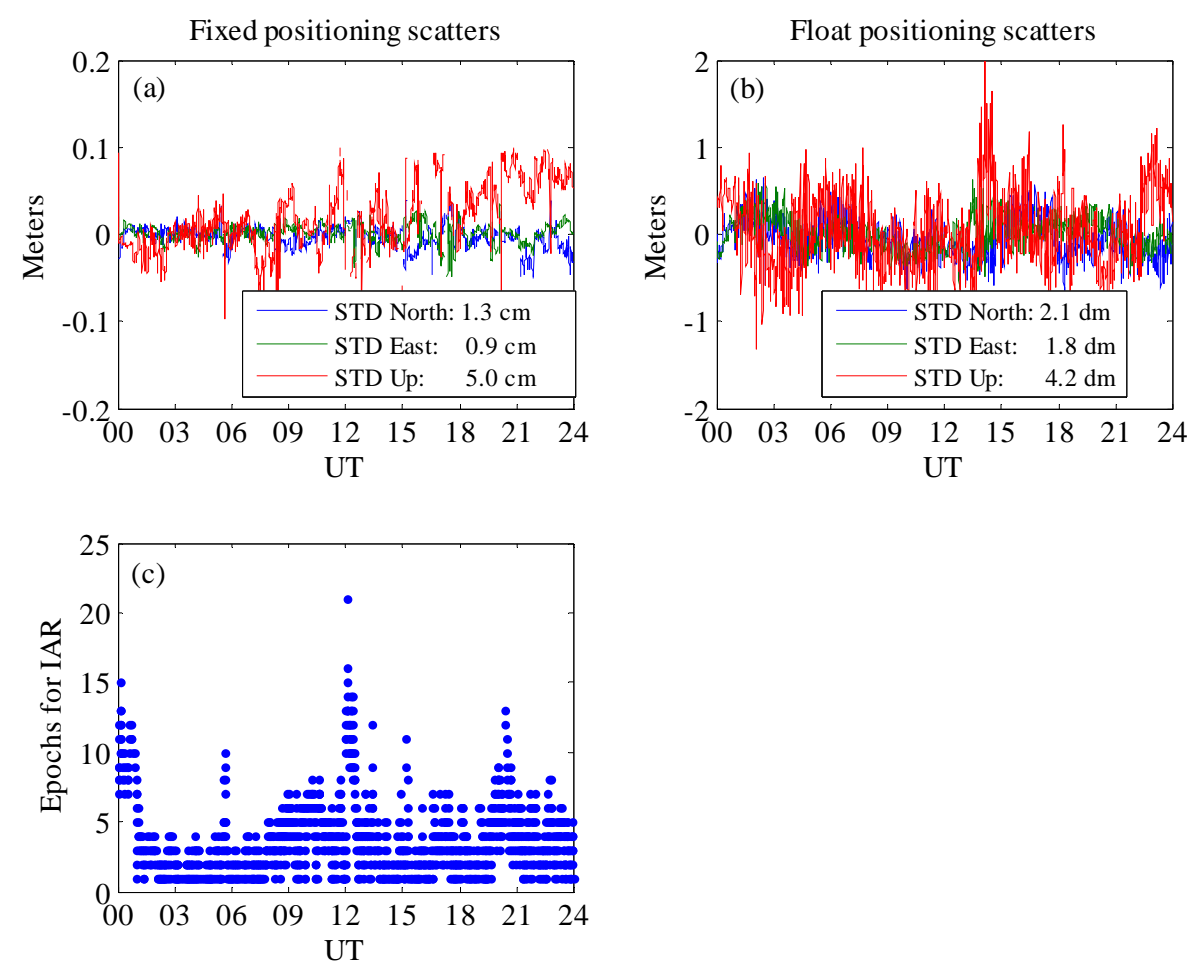

Figure 4. Dual-frequency PPP-RTK positioning for the user station BDAG within the northern China CORS network: panels (a) and (b): fixed \& float positioning scatters respectively; panel (c): number of epochs needed for successful IAR versus time of the day

Figure 4 shows the dual-frequency PPP-RTK performance at the BDAG location. The bottom panel indicates that the number of epochs needed for successful IAR is always less than 30 (15mins) and most of the time even under 10 (5mins). The corresponding accuracy of the ambiguity-fixed positioning (with respect to the known ground-truth) is about $1 \mathrm{~cm}$ and $5 \mathrm{~cm}$ for the horizontal and vertical components, respectively, while the accuracy of the ambiguity-float positioning is in the range of 2-4 dm.

3.2. Perth CORS: Single-frequency PPP. The Perth CORS network consists of 6 stations with inter-station distances in the range of 60-180 km, see Figure 5. The dual-frequency (L1-L2-C1-P2) Trimble NetR5 GPS data collected on 23rd October 2010 with the 30 sec sampling rate was selected for the test of the network processing, while the single-frequency (C1-L1) UBlox GPS data was used for the test of the user's PPP processing. The configuration of the network and the user station are displayed in Figure 5. The test results are shown in Figure 6. 


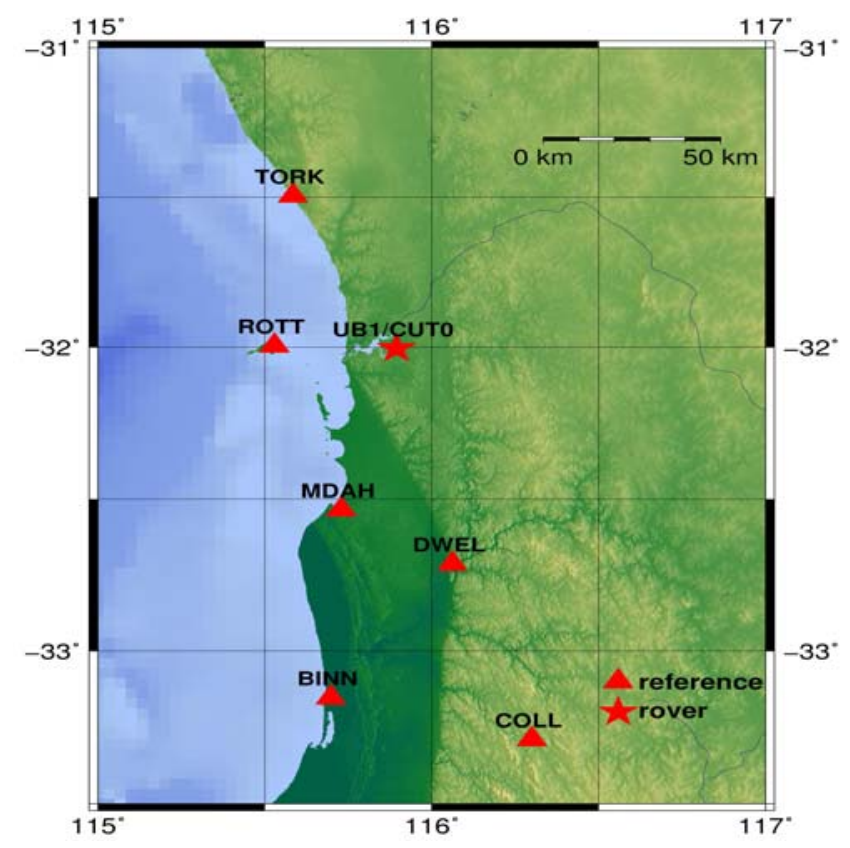

Figure 5. Configuration of the Perth CORS network (triangles) and the single-frequency PPP user: UB1 (star); the CUT0 is a dual-frequency receiver for forming a zero baseline with UB1
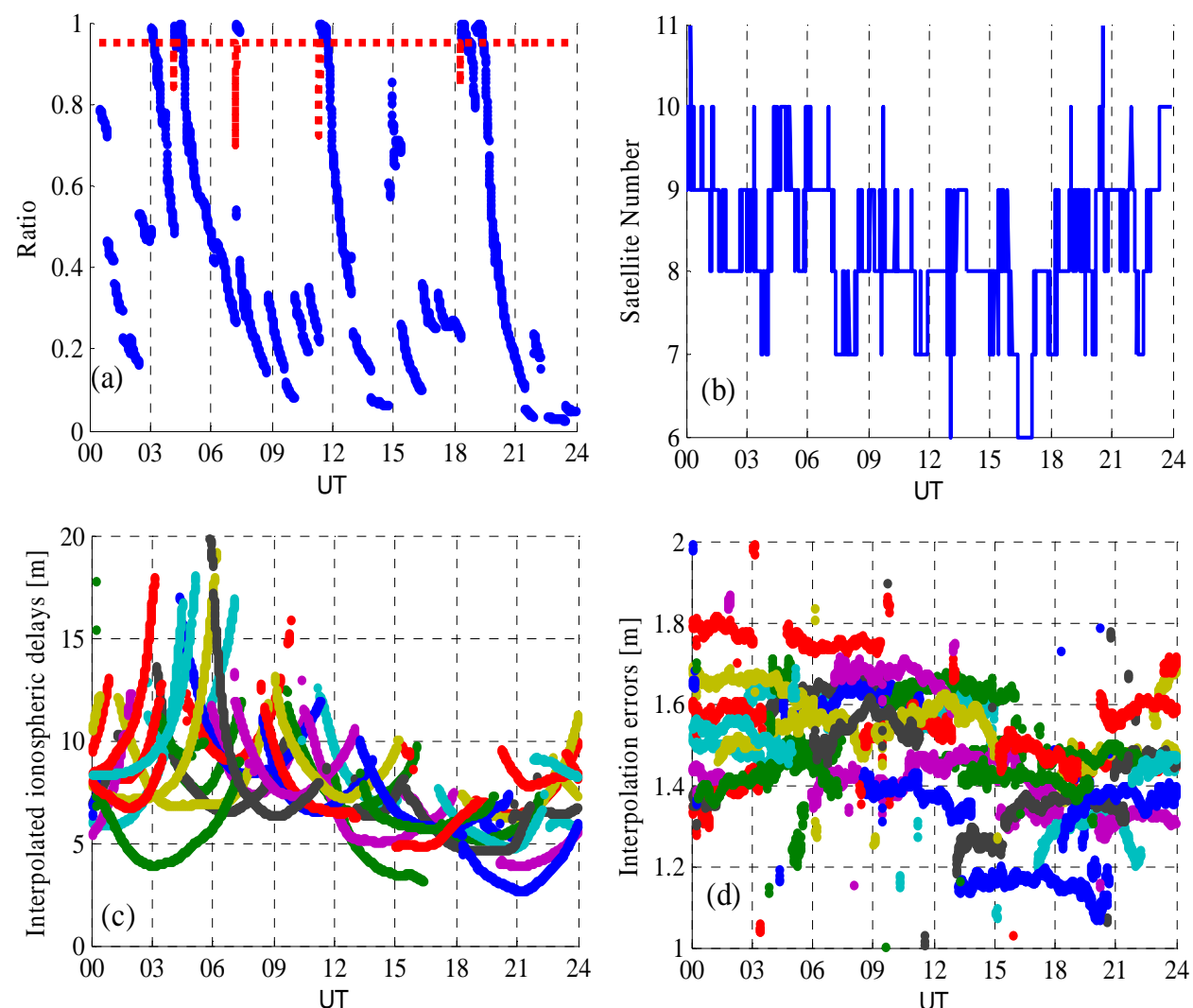

Figure 6. Perth network processing results: panel (a) FFRatio test- and threshold values versus time; panel (b) number of tracked satellites versus time; panels (c) \& (d) interpolated ionospheric delays at UB1 and their errors respectively. Different colors correspond to different satellites 
3.2.1. Perth Network Processing Results. For the network data processing, the same strategy as for the northern China network was used. Panel (a) in Figure 6 shows the results of ratio tests for network IAR with success rate roughly $2571 / 2782=92.4 \%$. The performance is slightly worse than that of the northern China network. This may be attributed to both the scale of the network and the increase in the number of ambiguities per epoch.

To weight out the performance of ionosphere interpolation, the reference ionospheric delays at UB1 are derived from post-processing the dual-frequency GPS data from the reference network and the CUT0. Panel (d) in Figure 6 shows the differencing values of the interpolated ionospheric delays and these references, from which the STD value calculated for the ionospheric interpolation precision is $1.4 \mathrm{dm}$ (Ciraolo et al, 2007).

3.2.2. Single-frequency PPP Results. The procedure and settings were similar as before, but with the standard deviation of the pseudo ionospheric observables set to $1.4 \mathrm{dm}$.
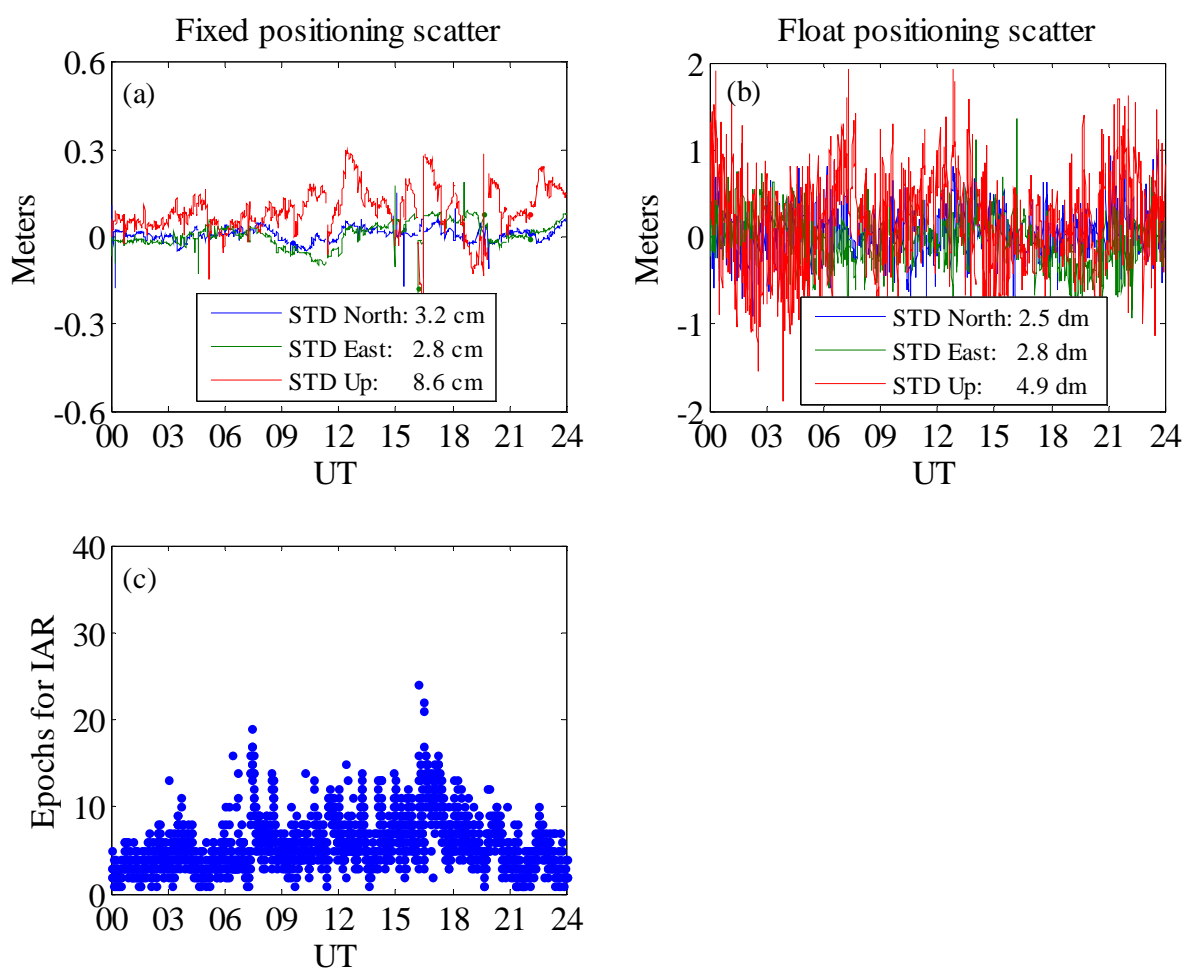

Figure 7. Results of PPP-RTK at the single-frequency UB1 station, each panel has the same meaning as those in Figure 4

Similar to Figure 4, Figure 7 shows the full IAR performance of the single-frequency PPP-RTK positioning. From the bottom panel, it can be seen that the maximum number of epochs needed for IAR in PPP is less than 40 (20mins) and the averaging number of epochs needed for IAR is about 10 (5mins). The accuracies of the ambiguity-fixed positioning in the horizontal component are in the range of 2-3 cm and in the vertical direction is less than $1 \mathrm{dm}$. In contrast, the accuracies of the ambiguity-float positioning in three components are in the range of 2-5 dm.

4. CONCLUSIONS. In this contribution, we described a novel PPP-RTK approach and demonstrate its potential for high accuracy positioning which is due to the realised PPP-user integer ambiguity resolution capabilities. To emphasise the flexibility of our approach, we also show the data processing methods for the two the eases: with and without satellite clock information provided externally. 
In our PPP-RTK approach the UD GNSS network observations are processed by solving a re-parameterised, full-rank system of observation equations. The re-parameterisation eliminates the system rank defect, thereby estimable parameters in the network can be obtained. These estimable parameters include the SD (biased) receiver clocks, the (biased) satellite clocks, the (biased) phase and code instrumental delays, the DD ambiguities, the SD ZTDs and the ionospheric model parameters. After network ambiguity is resolved, the PPP-user use the relevant ambiguity-fixed network parameters (e.g. biased satellite clock, satellite phase bias and interpolated ionospheric slant delay) in his own estimation procedure, which enables the PPP-user to perform integer ambiguity resolution and realise cm-level positioning. Our PPP-RTK concept combines the merits of both PPP and network-based RTK. Its performance is demonstrated by the tests of two CORS networks: one is a northern China network and the other is a Western Australia network near Perth.

\section{ACKNOWLEDGMENTS}

This work was conducted in the context of the Australian ASRP research project "Space Platform Technologies”. Data from the West Australian Network is kindly provided by GPS Network Perth. The authors thank two reviewers, Dr Suqin Wu and Prof. Xiufeng He, for their constructive comments. The second author is the recipient of an Australian Research Council (ARC) Federation Fellowship (project number FF0883188). All this support is gratefully acknowledged.

\section{REFERENCES}

Bree, R. J. P., Tiberius, C. C. J. M. and Hauschild, A. (2009). Real time satellite clocks in single frequency precise point positioning. Proceedings of the ION GNSS 2009, Savannah, Georgia, USA.

Ciraolo, L., Azpilicueta, F. J., Brunini, C., Meza, A. and Radicella, S. M. (2007). Calibration errors on experimental slant total electron content (TEC) determined with GPS. Journal of Geodesy, 81, 111-120.

De Jonge, P. J. (1998). A processing strategy for the application of the GPS in networks. Netherlands Geodetic Commission.

Geng, J., Teferle, F. N., Meng, X. and Dodson, A. H. (2010). Towards PPP-RTK: Ambiguity resolution in real-time precise point positioning. Advances in space research, doi:10.1016/j.asr.2010.03.030.

Jarlemark, P. O. J. and Emardson, T. R. (1998). Strategies for spatial and temporal extrapolation and interpolation of wet delay. Journal of Geodesy, 72, 350-355.

Kouba, J. and Heroux, H. (2001). Precise point positioning using IGS orbit and clock products. GPS Solutions, 5, $12-28$.

Li, X., Zhang, X. and Ge, M. (2010) Regional reference network augmented precise point positioning for instantaneous ambiguity resolution. Journal of Geodesy, 85, 151-158.

Odijk, D., Verhagen, S., Teunissen, P. J. G., Hernandez-Pajares, M., Juan, M. J., Sanz, J., Samson J. and Tossaint M. (2010). LAMBDA-Based Ambiguity Resolution for Next-Generation GNSS Wide Area RTK. Proceedings of the 2010 International Technical Meeting of the Institute of Navigation, San Diego, California, USA.

Sardon, E. and Zarraoa, N. (1997). Estimation of total electron-content using GPS data: how stable are the differential satellite and receiver instrumental biases? Radio Science, 32, 1899-1910.

Teunissen, P. J. G. (1995). The least squares ambiguity decorrelation adjustment: a method for fast GPS integer ambiguity estimation. Journal of Geodesy, 70, 65-82.

Teunissen, P. J. G. and Kleusberg, A. (1998). GPS for Geodesy, 2nd edition. Springer-Verlag.

Teunissen, P. J. G. and Verhagen, S. (2009). The GNSS ambiguity ratio-test revisited. Survey Review, 41, $138-151$.

Teunissen, P. J. G., De Jonge, P. J. and Tiberius, C. C. J. M. (1996). Volume of the GPS ambiguity search space and its relevance for integer ambiguity resolution. Proceedings of the 1996 9th International Technical Meeting of the Satellite Division of the Institute of Navigation, ION GPS-96. Part 1 (of 2), Kansas City, MO, USA.

Teunissen, P. J. G., Odijk, D. and Zhang, B. C. (2010). PPP-RTK: results of CORS network-based PPP with integer ambiguity resolution. Journal of Aeronautics, Astronautics and Aviation, 42, 223-230.

Vollath, U., Deking, A., Landau, H., Pagels, C. and Wagner, B. (2000). Multi-base RTK positioning using Virtual Reference Stations. Proceedings of the ION GPS 2000, Salt Lake City, Utah, USA.

Wu, S. Q., Zhang, K. F. and Silcock, D. (2008). An Investigation of Performance Difference of Regional Atmospheric Models for Network RTK-A Case Study in Victoria. Proceedings of International Symposium on GPS/GNSS 2008, Tokyo, Japan.

Wuebbena, G., Schmitz, M. and Bagge, A. (2005). PPP-RTK: Precise Point Positioning using state-space representation in RTK networks. Proceedings of the ION GNSS 2005, Long Beach, California, USA.

Yuan, Y. B. and Ou, J. K. (2001). An improvement on ionospheric delay correction for single frequency GPS user-the APR-I scheme. Journal of Geodesy, 75, 331-336.

Yuan, Y. B., Huo, X. L., Ou, J. K., Zhang, K. F., Chai, Y. J., Wen, D. B. and Grenfell, R. (2008a). Refining the Klobuchar ionospheric coefficients based on GPS observation. IEEE transactions on aerospace and electronic systems, 44, $1498-1510$.

Yuan, Y. B., Tscherning, C. C., Knudsen, P., Xu, G. C. and Ou, J. K. (2008b). The ionospheric eclipse factor method (IEFM) and its application to determining the ionospheric delay for GPS. Journal of Geodesy, 82, 1-8.

Zhang, K., Wu, F., Wu, S., Rizos, C., Roberts, C., Ge, L., Yan, T., Gordini, C., Kealy, A., Hale, M., Ramm, P., Asmussen, H., 
Kinlyside, D. and Harcombe, P. (2006). Sparse or dense: Challenges of Australian network RTK. Proceedings of International Global Navigation Satellite Systems Society IGNSS Symposium 2006, Queensland, Australia.

Zumberge, J., Heflin, M., Jefferson, D., Watkins, M. M. and Webb, F. H. (1997). Precise point positioning for the efficient and robust analysis of GPS data from large networks. Journal of Geophysical Research, 102, 5005-5017. 